\title{
BLUE LIGHT REDUCING SOFTWARE APPLICATIONS FOR MOBILE PHONE SCREENS: MEASUREMENT OF SPECTRAL CHARACTERISTICS AND BIOLOGICAL PARAMETERS
}

\author{
S. Mitropoulos', V. Tsiantos ${ }^{2}$, A. Americanos ${ }^{3}$, I. Sianoudis ${ }^{3}$, A. Skouroliakou' ${ }^{*}$ \\ ${ }^{1}$ Department of Biomedical Engineering, University of West Attica, Athens, Greece \\ 2Department of Physics, International Hellenic University, Thessaloniki, Greece \\ 3Department of Biomedical Sciences, University of West Attica, Athens, Greece
}

\begin{abstract}
The displays of the majority of electronic devices nowadays are illuminated by Light-Emitting Diodes (LEDs) or Organic Light-Emitting Diodes (OLEDs). These types of light sources have certain advantages regarding colour variety, contrast, resolution and the ability to construct thinner screens. Nevertheless, recent research raises concern of possible negative biological impact of these display types on visual health and the circadian rhythm. The biological basis of the concern lies in the emission spectra of the light sources. The white LEDs used as backlights in LED screens have a characteristic emission spectrum with a peak at $450 \mathrm{~nm}$ and the Red-Green-Blue (RGB) OLED emission spectrum has a blue peak. Both of them are very close to the 46onm where the melanopsin retina pigment presents the maximum absorption. In order to reduce the blue light emission several techniques have been developed including hardware adjustments, external filters and software applications that control the emission display characteristics. This study aims to record the performance of several available software applications on different mobile phone models. The spectral power distributions of the mobile phone screen were recorded by means of a commercial radiospectrometer, without and with the use of the blue light reducing software application, for various blue light filtering levels depending on the application. Several photometric and circadian parameters were calculated from the available spectra such as circadian light input, photopic illuminance and melatonin suppression index. The results of the study are the recordings of the respective differences in mobile screen output with and without the use of the blue light reduction application, presented in terms of spectral power and biologically relevant parameters. The analysis of the measuring procedure and the obtained results lead to an evaluation of the application performance variation depending on the mobile phone type and a standardised measurement protocol in order to have comparable results that could be used for blue light reducing software applications performance evaluation.
\end{abstract}

Keywords: Spectrophotometry, circadian parameters, blue light filtering

\section{INTRODUCTION}

Smartphones are part of our everyday life for communication, social and professional networking and leisure applications. Their use is well established and will probably increase in the near future. The visible light spectrum emitted by smartphone screens is mainly determined by screen technology. In most cases it is based on white LEDs whilst most sophisticated smartphone models use OLED based screens. In both cases the emitted light spectrum is characterized by three bands in the red, green and blue spectrum regions.

Apart from the visionary system light is also a significant input for the circadian regulation system. The human circadian clock organizes the timing of all daily biological functions and is regulated by the suprachiasmatic nuclei (SCN) in the hypothalamus. The temporal pattern of light and dark on the retina synchronizes the SCN to a 24 -h period matching to the earth's rotation rate on its axis [1]. The human visual and circadian systems are characterized by different mechanisms of light processing thus presenting different sensitivities and responses to retinal light exposure [2]. The visual system has a large dynamic response range covering 9-10 orders of magnitude. The response of the circadian system at optical radiation is characterized by a high threshold and quick saturation with a dynamic range of two orders of magnitude. The maximal sensitivity of the circadian system is at $460 \mathrm{~nm}$ whereas the visual system presents its maximum sensitivity at $555 \mathrm{~nm}$.

The biological time keeping system regulates day night rhythms of behavior, endocrine regulation, immune response and energy metabolism among others. The biological clock is influenced by external light [3]. A number of studies have dealt with the characteristics of light influence on the circadian rhythm. Duffy and Czeisler [4] found a dose dependent relationship with response of the circadian system. Glickman and Levin [5] suggested that low intensity levels and short durations seem to affect the circadian system more. The greater impact of light stimuli on the circadian rhythm is during the natural dark phase.

\footnotetext{
*kskourol@uniwa.gr
} 
Chronic light exposure during the evening causes a phase delay. The adaptation of the organism to light exposure is also observed; therefore, the effects are dependent on repetition. The spectrum of the emitted light is also critical according to the sensitivity of photoreceptors to different wavelengths. The spectral sensitivity of the circadian system is complex and not yet completely understood. Overall, the greatest sensitivity is observed at short wavelengths (46onm 490nm). While disturbance of the circadian rhythm can occur with any type of light, the effect is more profound when certain LEDs are used as light sources and their influence depends on the specific properties of the particular source.

A number of indices have been proposed to characterize light interaction with the human circadian system. These indices are calculated from a measurable quantity, usually spectral irradiance $\mathrm{E}(\lambda)$.

The human optical system response to light is characterized by $\mathrm{V} \lambda$, the photopic luminous efficiency function. Depending on the total irradiance, the photopic or scotopic luminous efficiency functions are used to convert radiometric to photometric units.

Studies on the impact of light at night regarding the synthesis of melatonin have shown a peak spectral sensitivity at 46onm [6], [7]. Melatonin, also called the sleeping hormone, is produced by the pineal gland and is released mainly during the night [8]. Two light variables, intensity and wavelength [9], are responsible for the suppression of melatonin and an illuminance of only 1,5 lux may disrupt circadian rhythms.

The melatonin suppression action spectrum (MSAS) was published in 2001 [6], [7] and represents to what extent each wavelength is efficient in suppressing melatonin production. The original dataset is quite small, ranging from $425 \mathrm{~nm}$ to $560 \mathrm{~nm}$. An extension from $380 \mathrm{~nm}$ to $730 \mathrm{~nm}$ was provided by Aube [8] who used a combination of two log normal curves to fit and extrapolate the original data.

Circadian stimulus (CS) and Circadian Light (CL) are calculated by a mathematical model implemented by Rea[1], based on the neuroanatomy and neurophysiology of the retina and on published psychophysical studies of nocturnal melatonin suppression. CL is essentially spectrally weighted irradiance for the human circadian system.

Special spectrophotometers based on the proposed interaction models have been developed and calibrated in order to measure light as it is perceived by the circadian system (Daysimeter, Actiwatch Spectrum)[10].

Research on the implementation of a comprehensive quantitative model of the interaction between light and the human circadian system is ongoing; nevertheless, two broad suggestions from the scientific community are the reduction of the unnecessary high levels of light at night and the attenuation of the short wavelength spectrum components.

To that end a number of smartphone software applications reduce the light emitted in the blue region of the spectrum by controlling the relative weight of the signal emitted by each color sub pixel, whilst maintaining the optimal illuminant quality.

Apple has embedded their Night Shift mode in iOS, within the quick access setting menu of their phones from September 2016. The Android operating system has an embedded Night Light feature in a number of its versions, while Samsung has a night mode setting called Blue Light Filter. A number of light filtering applications are also available for smartphone users.

\section{MATERIALS AND METHODS}

The Spectral Power Distribution (SPD) of the light emitted from the screen was assessed for a range of smartphones. The sample of the measured smartphones comprised six models operating with Android operating system and two models operating with iOS. The screen type was LTPS LCD or IPS LCD capacitive touchscreen. The blue light filtering was performed by the built-in filtering application (Night Shift, Night Light). For two Android smartphones a third-party filtering application was used and tweaked to mimic the built-in application settings.

Measurements were made using a modular (HR400oCG-UV-NIR) CCD array spectroradiometer (Ocean Optics, Inc., Dunedin, FL, USA), coupled by a metal jacketed optical fibre with a $400 \mu \mathrm{m}$ core diameter (Ocean Optics, Inc., Dunedin, FL, USA). The system was calibrated for spectral irradiance using a HL-3P-CAL tungsten-halogen lamp. A special black measuring case was constructed to ensure the standardized measuring position of the optical fibre, minimal reflectance of light and shielding from ambient light. The distance of the optical fibre from the smartphone screen was $5 \mathrm{~cm}$. Measurements were performed at the center of the screen, with an all-white background and brightness set at maximum. The first measurement was performed without the blue light filter and the second with the blue light reducing application on. Measurements were performed in the spectral range of $400 \mathrm{~nm}$ to $750 \mathrm{~nm}$ in terms of the absolute spectral irradiance $\left(\mu \mathrm{W} / \mathrm{cm}^{2}\right)$.

Raw spectral data were weighted with the photopic luminous efficiency function $\mathrm{V}_{\lambda}$ [3] and integrated to calculate illuminance. Circadian Stimulus (CS) and Circadian Light (CLa) were calculated from the application available from the Rensselaer Polytechnic Institute [11] web page.

$\mathrm{C}_{\lambda}$ index was calculated by integrating the weighted SPD with the function $c(\lambda)$. $c(\lambda)$ was polynomially interpolated from the data provided by Gall [12] which was based on the melatonin suppressing action data by Brainard and Thapan. Total spectrum and shortwave (SW) spectrum (420nm-500nm) power were calculated in radiometric and photometric units. Moreover, percentile reduction for all parameter values after the application of the blue light reduction filter was calculated. All processing was implemented with Octave software. 


\section{RESULTS}

The original measurements provide the Spectral Power Distribution in terms of irradiance (Fig. 1). The obtained original spectra are characterized by three broad peaks in the blue $(\sim 45 \mathrm{Onm})$, green $(\sim 53 \mathrm{Onm})$ and red $(\sim 630 \mathrm{~nm})$ wavelengths. The case presented in Figure 1 presents an interesting pattern of the spectrum in the red region where three distinct peaks are observed. The application of the blue light reduction filter selectively attenuates the short (blue) and medium (green) wavelengths whilst maintaining approximately the same irradiance value at higher (red) wavelengths. SPDs were weighted with the photopic luminous efficiency function $V_{\lambda}$, converted thus in photometric units (Fig. 2). $V_{\lambda}$ has the maximum weighting value at $555 \mathrm{~nm}$ where the human visual system presents its highest sensitivity.

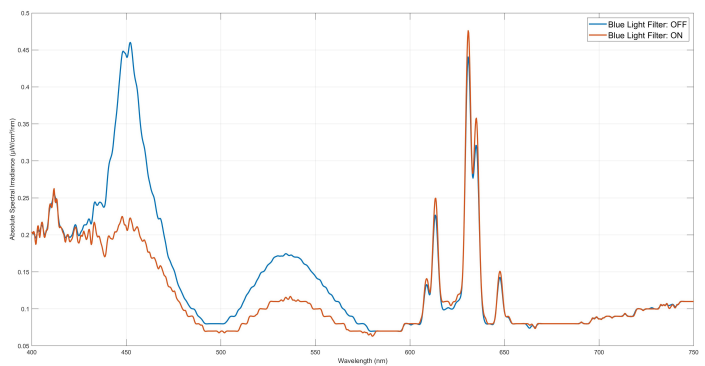

Figure 1. Spectral Power Distributions of a smartphone screen with the blue light filtering application off (blue line) and on (red line)

The reduction of the CS, CLa and $\mathrm{C}_{\lambda}$ parameters as well as total and SW spectrum power and their reduction after blue light filter application are presented in Figure 3.

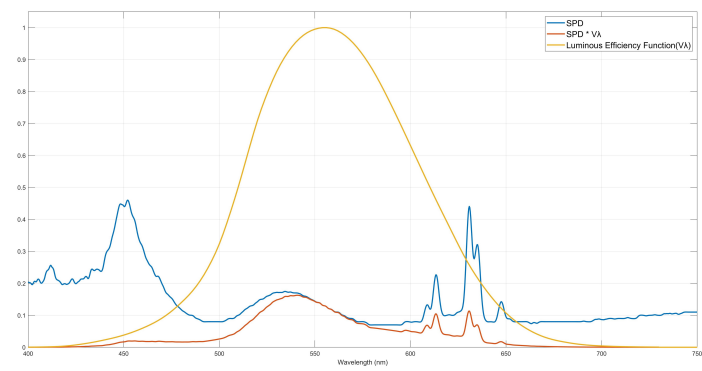

Figure 2. SPD in radiometric units, $\mathrm{V}_{\lambda}$ (photopic luminous efficiency function) and their product (spectral distribution in photometric units)

At the original SPDs with the brightness set to maximum the percentage of short wavelength light (420nm-50onm) was from $36 \%$ to $43 \%$. The corresponding SPDs with the blue light reduction application on, had a blue light percentage of $29.5 \%$ to $39.5 \%$. The application software led to a reduction of the blue light wavelengths from $12 \%$ to $31 \%$ depending on the smartphone model. Total irradiance (400nm$750 n m$ ) was reduced by $20 \%$ at the most.
Photobiological indices characterising circadian interaction with light were reduced from $15 \%$ to $30 \%$ when the filtering application was used.

\section{DISCUSSION}

Owing to the vast use of self-illuminating devices and the spectral characteristics of the light they emit, concern on the effects of short wavelengths to the human circadian system has risen during the past two decades. Research on the implementation of a model describing the interaction of visible electromagnetic radiation with the human circadian system is still ongoing. Certain photobiological parameters have been proposed by researchers and they are used to optimize conditions in everyday illuminating applications. As far as self-illuminating screens (computers, tablets, smartphones) are concerned, the reduction of short wavelength radiation is accomplished in three ways: external filters (glasses or screen covers), hardware adjustments (screen technologies) and software applications. Among the three options, software applications are the most convenient to use and can be applied to all smartphones.

In this study, the aim was to accurately measure and calculate radiometric, photometric and photobiological parameters in order to quantitatively evaluate the alterations in the emitted light spectrum of smartphone screens when a blue light filter software application was used.

All applications have proven effective in reducing the short wavelength spectral components. Their performance, however, does depend on the smartphone make and model. As expected, the reduction in the total spectrum power is higher in illuminance than in irradiance terms, whilst circadian indices variations correspond better with the SPD in radiometric terms.

Variations in the values of SPDs as well as photo biologically relevant parameters are high depending on the operating system and smartphone model. It should also be noted that the measurements were acquired with brightness set at maximum and full white background of the screen. The adjustment of brightness is available in smartphones and the circadian parameters would most likely be reduced with a lower brightness setting. The built in blue light filter application of the Android OS provides adjustment capabilities. Measurements were performed with the highest level of attenuation selected.

In general, up to $30 \%$ of reduction in photobiological indices can be accomplished by applying the filtering application. 


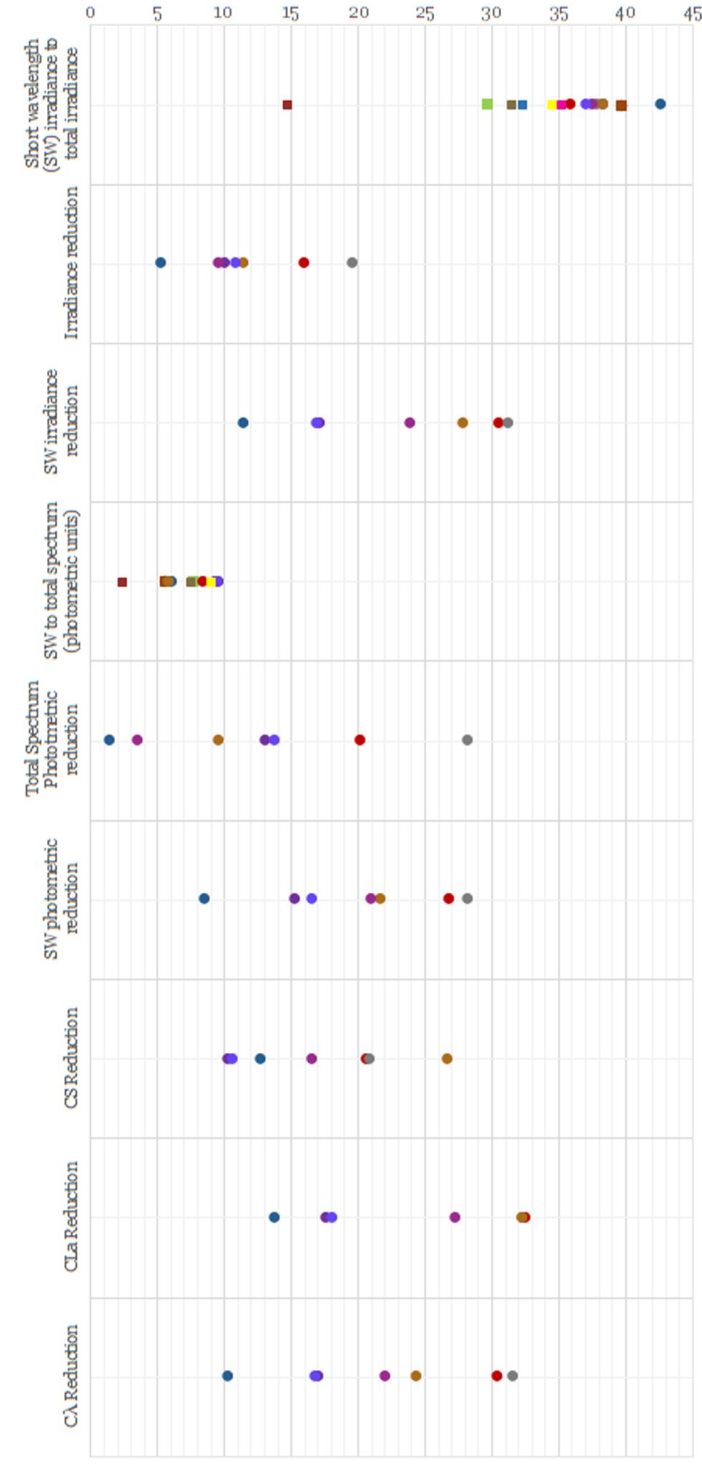

Figure 3. Percentile reduction in radiometric, photometric and circadian parameters (different colors correspond to different smartphone models)

\section{CONCLUSION}

As the blue light component seems to be affecting the circadian rhythm, it is advisable to adjust screen illumination characteristics according to the time of day. Built in applications in most cases prove very effective in selectively reducing shortwave visible electromagnetic radiation no matter which metric is used, up to $30 \%$. The spectral power distribution in radiometric terms seems more relevant in photobiologic calculations and should be measured in relevant experiments. Research on the exact quantitative interaction characteristics of the human circadian system and light is ongoing. However, smartphone users can effectively reduce their exposure to blue light maintaining good illuminating conditions by utilizing the available software applications.

\section{REFERENCES}

1. M. S. Rea, M. G. Figueiro, A. Bierman, J. D. Bullough, "Circadian light," J. Circadian Rhythms., vol. 8, no. 2, pp. 1 - 10, Feb. 2010.

DOI: $10.1186 / 1740-3391-8-2$

PMid: 20377841

PMCid: PMC2851666

2. M. G. Figueiro, R. Hamner, A. Bierman, M. S. Rea, "Comparisons of three practical field devices used to measure personal light exposures and activity levels," Ligh. Res. Technol., vol. 45, no. 4, pp. $421-434$, Aug. 2013.

DOI: $10.1177 / 1477153512450453$

PMid: 24443644 PMCid: PMC3892948

3. Opinion on Potential risks to human health of Light Emitting Diodes (LEDs), SCHEER, Brussels, Belgium, 2018.

Retrieved from:

https://ec.europa.eu/health/sites/health/files/scientifi c committees/scheer/docs/scheer o o 011.pdf

Retrieved on: Jul. 14, 2019

4. J. F. Duffy, C. A. Czeisler, "Effect of Light on Human Circadian Physiology," Sleep Med. Clin., vol. 4, no. 2, pp. $165-177$, Jun. 2009.

DOI: 10.1016/j.jsmc.2009.01.004

PMid: 20161220

PMCid: PMC2717723

5. G. Glickman, R. Levin, G. C. Brainard, "Ocular input for human melatonin regulation: relevance to breast cancer," Neuro Endocrinol. Lett., vol. 23, suppl 2: pp. 17 - 22, Jul. 2002.

PMid: 12163843

6. G. C. Brainard et al., "Action spectrum for melatonin regulation in humans: evidence for a novel circadian photoreceptor," J. Neurosci., vol. 21, no. 16, pp. $6405-$ 6412, Aug. 2001.

PMid: 11487664 PMCid: PMC6763155

7. K. Thapan, J. Arendt, D. J. Skene, "An action spectrum for melatonin suppression: evidence for a novel nonrod, non-cone photoreceptor system in humans," J. Physiol., vol. 535, no. 1, pp. $261-267$, Aug. 2001.

DOI: 10.1111/j.1469-7793.2001.to1-1-00261.x

PMid: 11507175

PMCid: PMC2278766

8. M. Aubé, J. Roby, M. Kocifaj, "Evaluating potential spectral impacts of various artificial lights on melatonin suppression, photosynthesis, and star visibility," PloS One, vol. 8, no. 7, pp. 1-15, Jul. 2013.

DOI: 10.1371/journal.pone.0067798

PMid: 23861808

PMCid: PMC 3702543

9. F. Falchi, P. Cinzano, C. D. Elvidge, D. M. Keith, A. Haim, "Limiting the impact of light pollution on human health, environment and stellar visibility," J. Environ. Manage., vol. 92, no. 10, pp. 2714 - 2722, Oct. 2011.

DOI: 10.1016/j.jenvman.2011.06.029

PMid: 21745709

10. M. S. Rea, M. G. Figueiro, "Light as a circadian stimulus for architectural lighting," Light. Res. Technol., vol. 50, no. 4, Dec. 2016.

DOI: $10.1177 / 1477153516682368$

11. Circadian stimulus calculator, Rensselaer Polytechnic Institute, Troy (NY), USA, 2018. 
Retrieved from: https://www.lrc.rpi.edu/cscalculator/ Retrieved on: Feb. 12, 2019

12. D. Gall, K. Bieske, "Definition and measurement of circadian radiometric quantities," in Proc. CIE Symp. '04: Light and Health, Vienna, Austria, 2004, pp. 129 132.

Retrieved from: http://www.cie.co.at/publications/ciesymposium-2004-light-and-health-non-visual-effects30-september-2-october-2004 Retrieved on: Apr. 11, 2019

13. J. Escofet, S. Bara, "Reducing the circadian input from self-luminous devices using hardware filters and software applications," Light. Res. Technol., vol. 49, no. 4, Dec. 2015.

DOI: $10.1177 / 1477153515621946$

14. L. T. Sharpe, A. Stockman, W. Jagla, H. Jägle, "A luminous efficiency function, $\mathrm{V}^{*}$ (lambda), for daylight adaptation," J. Vis., vol. 5, no. 11, pp. 948 - 968, Dec. 2005.

DOI: $10.1167 / 5.11 .3$

PMid: 16441195 